\title{
Molecular characterization and expression analysis of annexin B3 and B38 as secretory proteins in Echinococcus granulosus
}

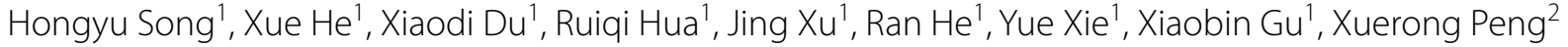
and Guangyou Yang ${ }^{1 *}$

\begin{abstract}
Background: Cystic echinococcosis is a parasitic zoonotic disease, which poses a threat to public health and animal husbandry, and causes significant economic losses. Annexins are a family of phospholipid-binding proteins with calcium ion-binding activity, which have many functions.

Methods: Two annexin protein family genes [Echinococcus granulosus annexin B3 (EgAnxB3) and EgAnxB38] were cloned and molecularly characterized using bioinformatic analysis. The immunoreactivity of recombinant EgAnxB3 (rEgAnxB3) and rEgAnxB38 was investigated using western blotting. The distribution of EgAnxB3 and EgAnxB38 in protoscoleces (PSCs), the germinal layer, 18-day strobilated worms and 45-day adult worms was analyzed by immunofluorescence localization, and their secretory characteristics were analyzed preliminarily; in addition, quantitative real-time reverse transcription polymerase chain reaction was used to analyze their transcript levels in PSCs and 28-day strobilated worms stages. The phospholipid-binding activities of rEgAnxB3 and rEgAnxB38 were also analyzed.

Results: EgAnxB3 and EgAnxB38 are conserved and contain calcium-binding sites. Both rEgAnxB3 and rEgAnxB38 could be specifically recognized by the serum samples from $E$. granulosus-infected sheep, indicating that they had strong immunoreactivity. EgAnxB3 and EgAnxB38 were distributed in all stages of E. granulosus, and their transcript levels were high in the 28-day strobilated worms. They were found in liver tissues near the cysts. In addition, rEgAnxB3 has $\mathrm{Ca}^{2+}$-dependent phospholipid-binding properties.

Conclusions: EgAnxB3 and EgAnxB38 contain calcium-binding sites, and rEgAnxB3 has $\mathrm{Ca}^{2+}$-dependent phospholipid-binding properties. EgAnxB3 and EgAnxB38 were transcribed in PSCs and 28-day strobilated worms. They were expressed in all stages of E. granulosus, and distributed in the liver tissues near the hydatid cyst, indicating that they are secreted proteins that play a crucial role in the development of E. granulosus.
\end{abstract}

Keywords: Echinococcus granulosus, Annexin, Calcium ion, Western blotting, Immunofluorescence localization, Secretory protein

*Correspondence: guangyou1963@aliyun.com

1 Department of Parasitology, College of Veterinary Medicine, Sichuan Agricultural University, Wenjiang 611130, China

Full list of author information is available at the end of the article

\section{Background}

Cystic echinococcosis (CE), which is caused by the larval stage of Echinococcus granulosus, is a parasitic zoonosis currently prevalent in Mediterranean countries, southern South America, Central Asia, China, Australia and parts of Africa [1,2]. CE seriously threatens public health and impedes the development of animal husbandry, resulting in approximately US $\$ 3$ billion losses to the livestock

c) The Author(s) 2021. This article is licensed under a Creative Commons Attribution 4.0 International License, which permits use, sharing, adaptation, distribution and reproduction in any medium or format, as long as you give appropriate credit to the original author(s) and the source, provide a link to the Creative Commons licence, and indicate if changes were made. The images or other third party material in this article are included in the article's Creative Commons licence, unless indicated otherwise in a credit line to the material. If material is not included in the article's Creative Commons licence and your intended use is not permitted by statutory regulation or exceeds the permitted use, you will need to obtain permission directly from the copyright holder. To view a copy of this licence, visit http://creativeco mmons.org/licenses/by/4.0/. The Creative Commons Public Domain Dedication waiver (http://creativecommons.org/publicdomain/ zero/1.0/) applies to the data made available in this article, unless otherwise stated in a credit line to the data. 
industry each year [3]. It has been listed as one of the neglected tropical diseases by the World Health Organization $[4,5]$. E. granulosus pathogenic research is of great significance, and studying the mechanism of the interaction between $E$. granulosus and its host is also necessary for the prevention and control of CE.

Proteomics analysis revealed that annexins were present in E. granulosus hydatid fluid, protoscoleces (PSCs) culture fluid and exosomes [6-8]. Annexins are a family of phospholipid-binding proteins with calcium ionbinding activity, which function in calcium signaling, and are widely distributed in eukaryotic cells [9]. According to differences in gene location and protein structure, annexins are divided into five categories [10]. Annexins have multiple functions, such as cell anti-inflammatory activity, membrane repair and membrane transport, and probably participate in cell proliferation, differentiation, and apoptosis [11-15].

Parasite annexins have been found to regulate the host immune response and maintain the structural integrity of the cell $[16,17]$. Parasite annexins have also been considered as potential targets for the development of drug and vaccine candidates $[16,18]$. Leishmania promastigotes could combine annexin $V$ to ensure normal function in the absence of phosphatidylserine [19]. Annexin 2 of Schistosoma mansoni is involved in epidermal development [20]. Recombinant annexin B30 of S. mansoni gave no significant protection against the parasite, which suggested it may not be suitable as a vaccine candidate [21]. Annexin B1 of Taenia solium can downregulate the immune response of the host $[22,23]$. In humans, E. granulosus annexins have only been found in the fluid of pulmonary hydatids, not in the fluid of vertebral or paravertebral hydatids, which suggests that they play an important role in the calcification process of cysts [7]. Up until now, only E. granulosus annexin B33 (EgAnxB33) has been studied [24]. In the present study, cloning, expression, bioinformatic analysis, western blotting, relative fluorescence quantitative polymerase chain reaction (PCR), immunofluorescence localization, and phospholipid-binding bioactivity analyses of $E g A n x B 3$ and $E g A n x B 38$ were performed to provide basic information and new research directions for the interaction between E. granulosus and its hosts.

\section{Methods}

\section{Animals}

Four female New Zealand rabbits (9 weeks old) were purchased from Dashuo Experimental Animal (Chengdu, China; experimental animal production license no. SYXK2019-189). Four male beagles (6 months old) were provided by the Dujiangyan Beagle Breeding Center of the Sichuan Institute of Musk Deer Breeding.
Albendazole and levamisole were used for deworming in the first 3 months.

\section{Parasites}

Cysts of E. granulosus were obtained from naturally infected sheep in Sichuan Province, China. PSCs and the germinal layer were separated aseptically as described previously $[25,26]$. The 28 -day strobilated worms were acquired by artificially infecting beagles. Each beagle was given 50,000 PSCs orally and then euthanized after 28 days. The 18-day strobilated worms and 45-day adult worms were provided by the Department of Parasitology of Sichuan Agricultural University.

\section{Sera}

Sera against E. granulosus were isolated from naturally infected sheep. Negative sera were collected from cestode-free sheep. Corresponding sera were obtained in Sichuan Province, China and infection was determined by autopsy.

The preparation process of the polyclonal antibodies of recombinant $E g A n x B 3(r E g A n x B 3)$ and $r E g A n x B 38$ was the same as previously reported [26]. Briefly, rabbit sera were collected as the negative control before immunization. Then each rabbit was immunized with $200 \mu \mathrm{g}$ recombinant protein emulsified with Freund's complete adjuvant (Sigma-Aldrich, St. Louis, MO), followed by three boosters using Freund's incomplete adjuvant. Two weeks after the final immunization, antisera were isolated and immunoglobulin G (IgG) was extracted.

\section{Cloning of EgAnxB3 and EgAnxB38}

An RNA extraction kit (Tiangen, Beijing, China) was used to extract total RNA from PSCs, and the Reverse Transcription System Kit (TaKaRa, Dalian, China) was used to synthesize cDNA. Specific primers were designed at GenBank using the sequences of $E g A n \times B 3$ and EgAnxB38 (accession numbers XM_024495323.1 and XM_024494485.1, respectively). The sequence of EgAnxB3 was amplified using the primers $5^{\prime}$-CGGA TCCATGGCGACTGTCAAGCCTTGCTG-3' (BamHI) and $5^{\prime}$-CCGGAATTCTTACTCCAGTATGGCAAGC-3' (EcoRI). The sequence encoding EgAnxB38 was amplified using primers 5'-CGGATCCATGGCTGGCTAT CCTCCACC-3' (BamHI) and 5'-CGGAATTCTCAGGG TCCAACCAAAGCCAC-3' (EcoRI). Target fragments were amplified and cloned. Single colonies were selected for PCR identification, and the plasmid from the bacterial solution that tested positive by PCR was sequenced.

\section{Bioinformatic analysis}

The physicochemical properties were predicted using the Expasy proteomics server (http://au.expasy.org). The 
open reading frames (ORFs) of $E g A n x B 3$ and $E g A n x B 38$ were analyzed using ORF Finder (https://www.ncbi.nlm. nih.gov/orffinder/). Signal peptides and transmembrane area were predicted using online software SignalP (http:// www.cbs.dtu.dk/services/SignalP-3.0/) and TMHMM2.0 (http://www.cbs.dtu.dk/services/TMHMM-2.0/). Tertiary (three-dimensional) structures were modeled through SWISS-MODEL (http://swissmodel.expas y.org/). MEGA software (version 5.05) was used to construct the phylogenetic tree using the maximum likelihood method [27].

\section{Expression and purification of recombinant EgAnxB3 and EgAnxB38}

The correctly sequenced $E g A n x B 3$ and $E g A n x B 38$ plasmids were digested with restriction enzymes, ligated into the pET32a $(+)$ plasmid. The resulting recombinant plasmids were transformed into Escherichia coli BL21 (DE3) (Tiangen, Beijing). E. coli cells containing pET32aEgAnxB3 and pET32a-EgAnxB38 were cultivated at $37^{\circ} \mathrm{C}$ for $8 \mathrm{~h}$. Then the transformants were induced with $1 \mathrm{mM}$ isopropyl $\beta$-D-1-thiogalactopyranoside. The recombinant proteins were purified using a $\mathrm{Ni}^{2+}$ affinity chromatography column (Bio-Rad, Hercules, CA).

\section{Western blotting}

Western blotting was performed as described previously [28]. Briefly, the crude protein extracts of PSCs and the purified recombinant protein were transferred onto a polyvinylidene difluoride membrane. The membrane was incubated with sera [1:200 volume/volume (v/v) dilution] from CE-infected sheep or polyclonal antibodies (1:200 $\mathrm{v} / \mathrm{v}$ dilution) for $12 \mathrm{~h}$ at $4{ }^{\circ} \mathrm{C}$. After four washes, horseradish peroxidase (HRP)-conjugated sheep anti-rabbit IgG or rabbit anti-sheep IgG (1:2000 v/v dilution; Boster, Wuhan, China) was added and incubated for $1 \mathrm{~h}$ at $37^{\circ} \mathrm{C}$. The immunoreactive protein signals were visualized using an Enhanced HRP-DAB Chromogenic Substrate Kit (Tiangen).

\section{Quantitative real-time reverse transcription PCR}

Total RNA and cDNA of PSCs and 28-day strobilated worms were obtained as described above. Quantitative real-time reverse transcription PCR was used to analyze expression profiles of $E g A n \times B 3$ and $E g A n \times B 38$ in PSCs and 28-day strobilated worms. The primers for EgAnxB3 were 5'-TGCCAACACGGATGCCCAAAC-3' and $5^{\prime}$-CTGGTGCGGTGTGCGAGAAC-3'. The primers for $E g A n x B 38$ were 5'-CGCTACGCAGAGGAC AAGAACG-3' and 5'-CTCGCATCTACCCAGCAC CAAC- $3^{\prime}$. Expression of the actin gene was detected for use as an internal control for normalization. Primers specific to E. granulosus actin were $5^{\prime}$-ATGGTTGGT
ATGGGACAAAAGG- $3^{\prime}$ and $5^{\prime}$-TTCGTCACAATA CCGTGCTC- $3^{\prime}$. The data were analyzed using the $2^{-}$ $\Delta \Delta \mathrm{CT}$ method [29].

\section{Immunofluorescence localization}

Immunofluorescence localization was performed as described previously [26]. The sections (PSCs, germinal layer from fertile/infertile cysts, 18-day strobilated worms and 45-day adult worms) were incubated with purified anti-rEgAnxB3/anti-rEgAnxB38 rabbit IgG or negative rabbit sera (1:200 v/v dilutions) for about $14 \mathrm{~h}$ at $4{ }^{\circ} \mathrm{C}$. Fluorescein isothiocyanate-conjugated sheep anti-rabbit IgG (1:100 dilution in $0.1 \%$ Evans blue solution) was incubated with sections for $1 \mathrm{~h}$ at $37^{\circ} \mathrm{C}$ in the dark. The fluorescence signals were observed under a fluorescence microscope. Meanwhile, to detect the possible secretion of $E g A n x B 3$ and $E g A n x B 38$, the liver tissues away from the cysts and near the cyst wall were also evaluated [24].

\section{Phospholipid-binding bioactivity assay}

The preparation of liposomes was based on previous reports with some modifications [24, 30]. Soybean lecithin (0.9 g; Sangon, Shanghai, China) and 0.3 g cholesterol (Sangon) were mixed and dissolved in anhydrous ethanol in a small beaker; the beaker was placed in a $65-70{ }^{\circ} \mathrm{C}$ water bath with stirring in order that the contents could dissolve completely, after which the beaker was rotated to remove the ethanol. Thirty milliliters of preheated phosphate-buffered saline was added to the beaker containing lecithin and cholesterol lipid membrane, and the contents stirred and heated in a water bath at $65-70{ }^{\circ} \mathrm{C}$ for $10 \mathrm{~min}$. Finally, the beaker was placed on a magnetic stirrer and the contents stirred for 30-60 min. The phospholipid-binding assay was performed as described previously $[24,31]$. There were three experimental groups for each recombinant protein: A, B, and $\mathrm{C}$. Twenty microliters of liposomes, $30 \mu \mathrm{r} E g \mathrm{AnxB} 3 /$ $\mathrm{r} E g \mathrm{AnxB} 38$, and $30 \mu \mathrm{l}$ of $1 \mathrm{mM} \mathrm{CaCl}{ }_{2}$ (except for group C) was added to each group, and $50 \mathrm{mM}$ Tris- $\mathrm{HCl}$ added as a supplement to a total volume of $100 \mu \mathrm{l}$. All the groups were incubated in ice water and centrifuged to separate the supernatant from the precipitate. The precipitate in group B was washed with Tris- $\mathrm{HCl}$. Thirty microliters of $1 \mathrm{mM}$ ethylenediaminetetraacetic acid (EDTA) and $70 \mu \mathrm{l}$ of Tris- $\mathrm{HCl}$ was added to the precipitate of group $\mathrm{B}$ and the mixture incubated in ice water for $30 \mathrm{~min}$. The supernatant and precipitate were separated by centrifugation. All the supernatant and precipitate samples were analyzed using $12 \%$ sodium dodecyl sulphate-polyacrylamide gel electrophoresis. 


\section{Results}

\section{Gene amplification and bioinformatics analysis}

The results of the bioinformatics analysis of EgAnxB3 and $E g A n x B 38$ are shown in Table 1. Both EgAnxB3 and EgAnxB38 were predicted to be extracellular proteins, but without a signal peptide.

Homology modeling was carried out on EgAnxB3 and EgAnxB38 in the SWISS-MODEL database, and two proteins with high homology were found, whose Protein Data Bank numbers were 1ala.1.A and 1aii.1.A. Using 1ala.1.A and 1aii.1.A as templates, three-dimensional models of EgAnxB3 and EgAnxB38 were constructed, respectively. The similarity between $E g \mathrm{AnxB} 3$ and the template sequence was $45.75 \%$, while the similarity was $39.31 \%$ between $E g A n x B 38$ and the template sequence. Both EgAnxB3 and EgAnxB38 have calciumbinding sites, but EgAnxB3 contains two types of calcium ion-binding domain, whereas EgAnxB38 only contains one calcium ion-binding domain (Fig. 1).
DNAMAN was used to compare the protein sequences of $E g A n x B 3$ and $E g A n x B 38$, and it was found that the similarity was $32.15 \%$. Multiple sequence alignment revealed that EgAnxB3 shared $97.42 \%$ identity with Echinococcus multilocularis annexin, $83.23 \%$ with T. solium AnxB3, and $63.87 \%$ with Hymenolepis microstoma annexin (Fig. 2a). EgAnxB38 shared 92.63\% identity with E. multilocularis annexin, $64.13 \%$ with $H$. microstoma annexin, $40.17 \%$ with Fasciola hepatica annexin, and $39.88 \%$ with Clonorchis sinensis annexin (Fig. 2b). The maximum likelihood phylogenetic tree demonstrated that $E g A n x B 3$ and $E g A n x B 38$ were located on different branches, and had the closest genetic relationship with $E$. multilocularis (Fig. 3).

\section{Expression, purification, and western blotting of $r E g A n \times B 3$ and $\mathrm{rEgAnxB38}$}

The molecular mass of EgAnxB3 was about $52 \mathrm{kDa}$, which was close to the expected value [note: the pET$32 \mathrm{a}(+)$ tag proteins weigh about $18 \mathrm{kDa}$. Solubility

Table 1 Bioinformatics analysis of Echinococcus granulosus annexin B3 (EgAnxB3) and EgAnxB38

\begin{tabular}{llllll}
\hline & Open reading frame & Amino acid & $\begin{array}{l}\text { Molecular weight } \\
(\mathrm{kDa})\end{array}$ & $\begin{array}{c}\text { Isoelectric point (pl) } \\
\text { coefficient }\end{array}$ & $\begin{array}{c}\text { Lipid solubility } \\
\text { coefficient }\end{array}$ \\
\hline EgAnxB3 & 933 & 310 & 34.8 & 5.37 & 32.55 \\
EgAnxB38 & 1356 & 451 & 48.5 & 6.66 & 33.45 \\
Instability
\end{tabular}

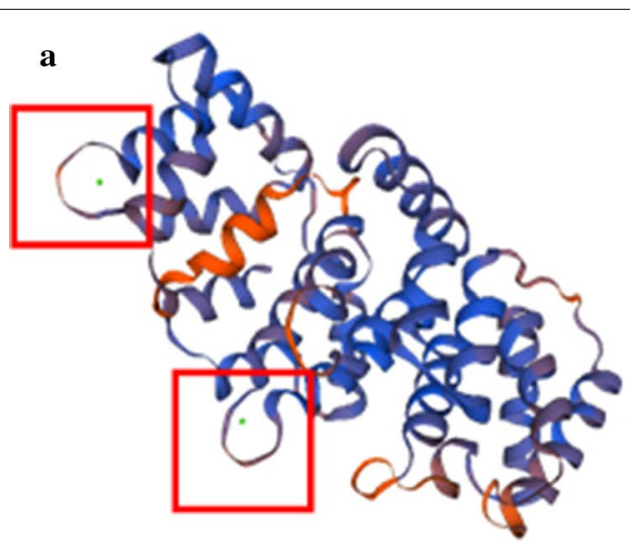

b

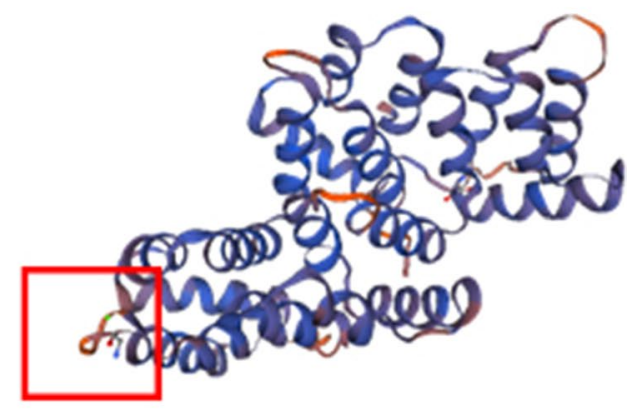

Fig. 1 Three-dimensional structure model prediction of Echinococcus granulosus annexin B3 (EgAnxB3) (a) and EgAnxB38 (b). The red box shows the calcium ion-binding area

(See figure on next page.)

Fig. 2 Sequence alignment of EgAnxB3 (a) and EgAnxB38 (b). Note the K-G-X-G-T sequence, indicated by yellow arrows. a Eg Echinococcus granulosus [GenBank (gb) XP_024350278.1], Em Echinococcus multilocularis (gb CDI98572.1), Ts Taenia solium (gb AAY27744.1), Hm Hymenolepis microstoma (gb CDS27549.1), Hs Homo sapiens (gb CAG46637.1), Sm Schistosoma mansoni (gb AAC79802.3), Fh Fasciola hepatica (gb THD28189.1), Sj Schistosoma japonicum (gb TNN13360.1). b Eg E. granulosus (gb CDS24484.1), Em E. multilocularis (gb CDS35601.1), Hm Hymenolepis microstoma (gb CDS26802.2), Fh F. hepatica (gb THD25992.1), Cs Clonorchis sinensis (gb RJW68544.1), Ov Opisthorchis viverrini (gb OON18674.1), Sh Schistosoma haematobium (gb XP_012796077.1), Hs Homo sapiens (gb AAH00871.1); for other abbreviations, see Fig. 1 

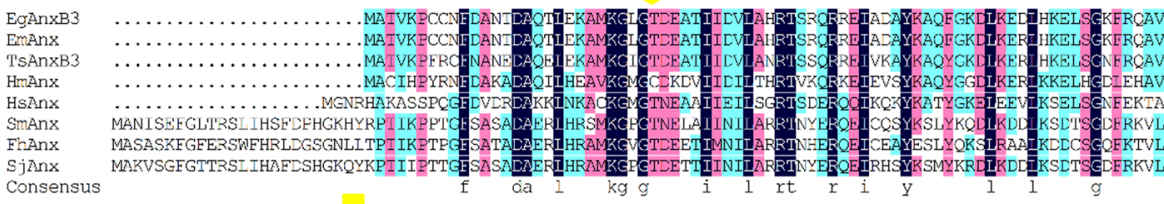

\section{ENSFYNRNHVNA}

TsAnxB3

$\operatorname{Hrm} \ln x$

HSAnx

FimAnx

SjAnx

EWSLYDRAHVNA

LWSFI TPNEINAI

CQI TVDTFYMT A

Consensu

COI WDTEYMI A1

EgọnxB3

Emanx

Tsanxis

Hrminx

HisAnx

FhAnx

SjAnx

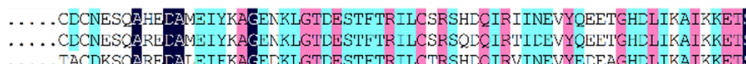

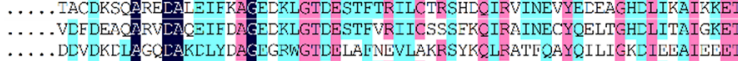

......DEVDKLLA

DKGVNSIINHEI DEA LAKLLYASGAGRVGISERRITRVICNRTPYCLYLTSEIYFKMYGKTLIEHIESE

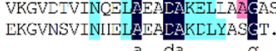

SRVGTNEKRITRVICGRTPECLRAANDAYAKLFGKSLLLDNTSE

SEISGL

RKT YLLVRCAODCELY

IRKIOAKIDSTYEKSIVKMISGDTSGCYKNILLAILE. . . . .

$\operatorname{Ranhx}$

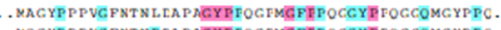

egmexro

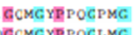

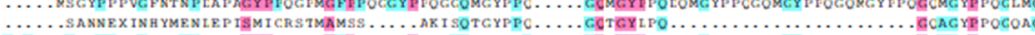

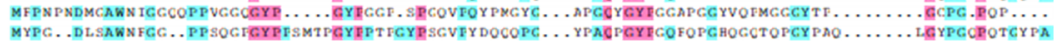

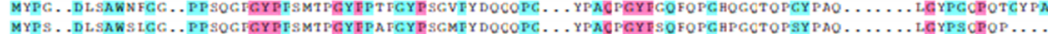

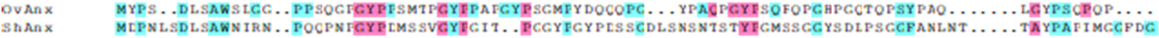

$\operatorname{shanx}$

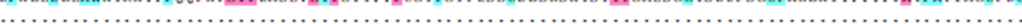
Consensu

Eg An $x$

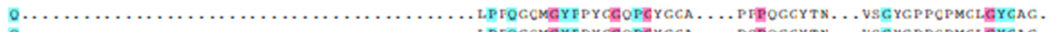

$\operatorname{Ran} \operatorname{An} x$

PhAn

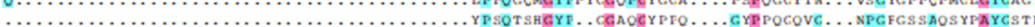

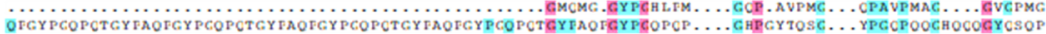

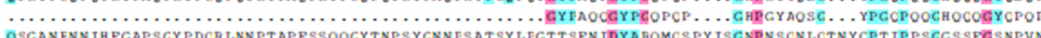

sis

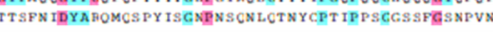

EqAn $\times$ B3 B

AVAGPSGSCGEGAMMGPLSIYYSGR.

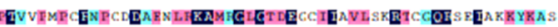
AVAGPSGSCSGCAMMCPLS IY YSGR.

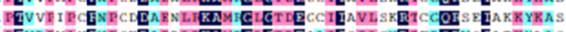

$\operatorname{Ran} \operatorname{An} x$ OTLGPIPNYGE...MGGISAYYSGR.

$\operatorname{cosinx}$ GYSPMCOATGG.

$\operatorname{Oran} x$ CYSPMCGATOG. .TSFVGAAPRES.

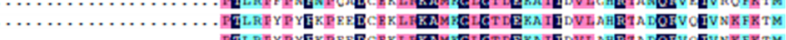

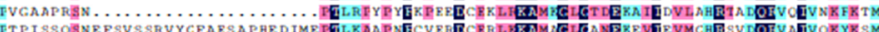

$\sin x$

Consenatua MAS I WVG HEG

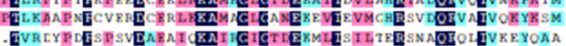

F $9 \operatorname{An} \times B$

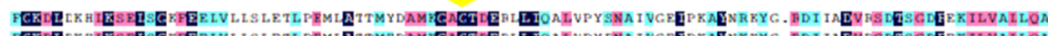

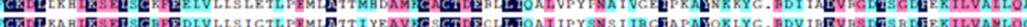

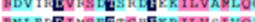

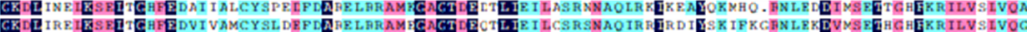

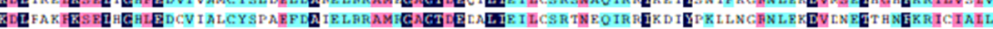

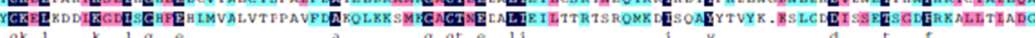

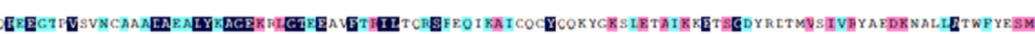

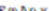

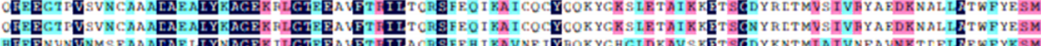

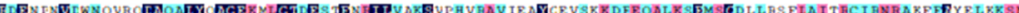
W

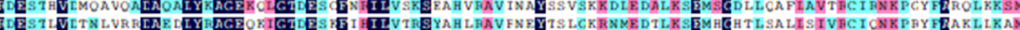

$\sin \sin x$

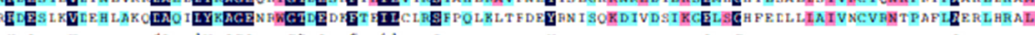

E.gAn $\times$ Bз3

$\operatorname{Emanx}$

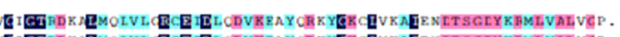

$\tan \operatorname{An} x$ IGT

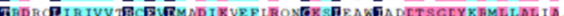

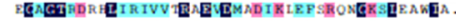

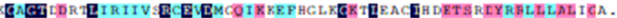

$\tan \sin x$

Identity

$92.63 \%$

$40.17 \%$

$39.35 \%$

$27.85 \%$ 


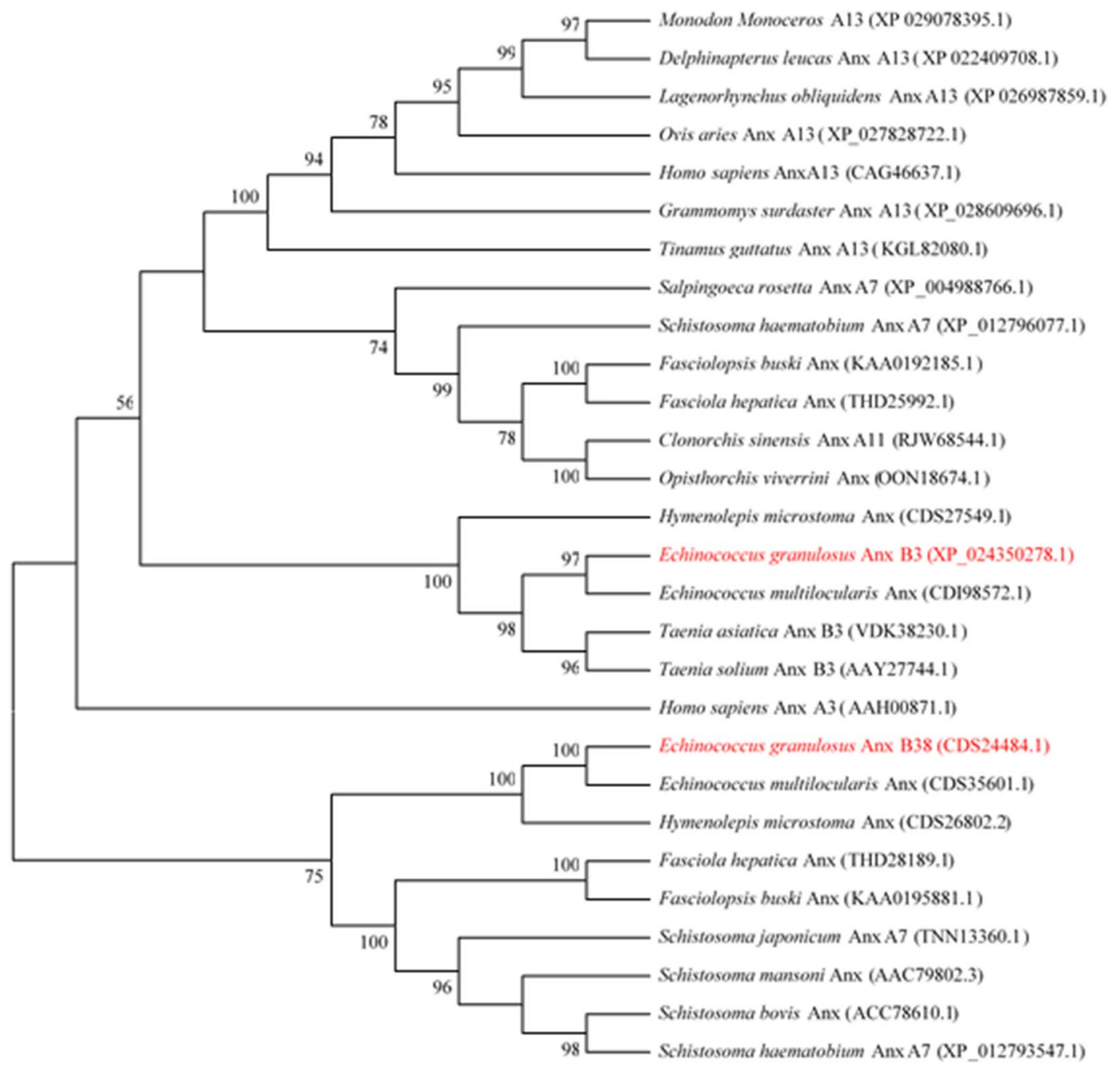

Fig. 3 Maximum likelihood phylogenetic tree of EgAnxB3 and EgAnxB38. For abbreviations, see Fig. 1

analysis showed that $E g A n x B 3$ was largely expressed as a soluble form and partly in inclusion bodies (Fig. 4a). The molecular mass of EgAnxB38 was about $67 \mathrm{kDa}$. Solubility analysis showed that EgAnxB38 was largely expressed in inclusion bodies (Fig. 4b). Western blotting showed that $\mathrm{r} E g \mathrm{AnxB} 3$ and $\mathrm{r} E g \mathrm{AnxB} 38$ reacted with rabbit anti$\mathrm{r} E g$ AnxB3 antibody and rabbit anti-rEgAnxB38 antibody, respectively, and with CE-positive sheep sera. Moreover, the native $E g A n x B 3$ protein and $E g A n x B 38$ protein in crude protein from PSCs were recognized using the rabbit anti-rEgAnxB3 antibody and rabbit anti-rEgAnxB38 antibody, respectively (Fig. 4).

\section{Transcriptional profiles of EgAnxB3 and EgAnxB38}

$E g A n \times B 3$ and $E g A n \times B 38$ genes were transcribed in both PSCs and 28-day strobilated worms, and the transcript levels of EgAnxB3 and EgAnxB38 in 28-day strobilated worms were both significantly higher $(P<0.05)$ than those in the PSCs (Fig. 5).

\section{Immunolocalization of EgAnxB3 and EgAnxB38}

$E g A n x B 3$ and $E g A n x B 38$ were distributed in the germinal layer of fertile/infertile cysts, the parenchyma and tegument of 18-day strobilated worms and 45-day adult worms, and the calcareous corpuscles and hooks of PSCs. Compared with EgAnxB3, EgAnxB38 was not only distributed in the calcareous corpuscles and hooks of PSCs, but also in the tegument of PSCs. Meanwhile, the distribution range of $E g A n x B 38$ in fertile/infertile cysts, adult worms, and PSCs was significantly wider than that of $E g A n x B 3$, showing a strong fluorescence signal (Fig. 6).

$E g A n x B 3$ and $E g A n x B 38$ were distributed in hepatic sinusoids of liver tissue close to hydatid cysts. In addition, $E g A n x B 38$ was also found in hepatic sinusoids in the 


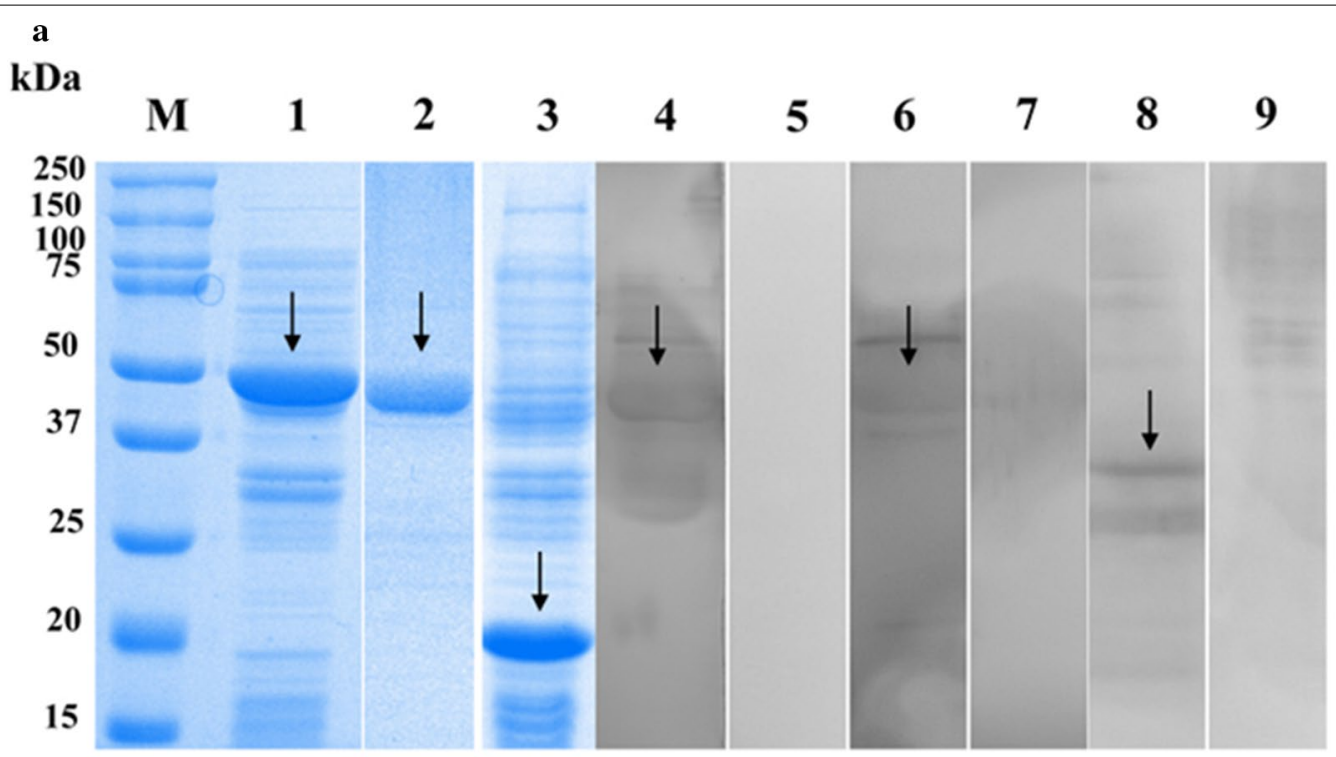

\section{b}

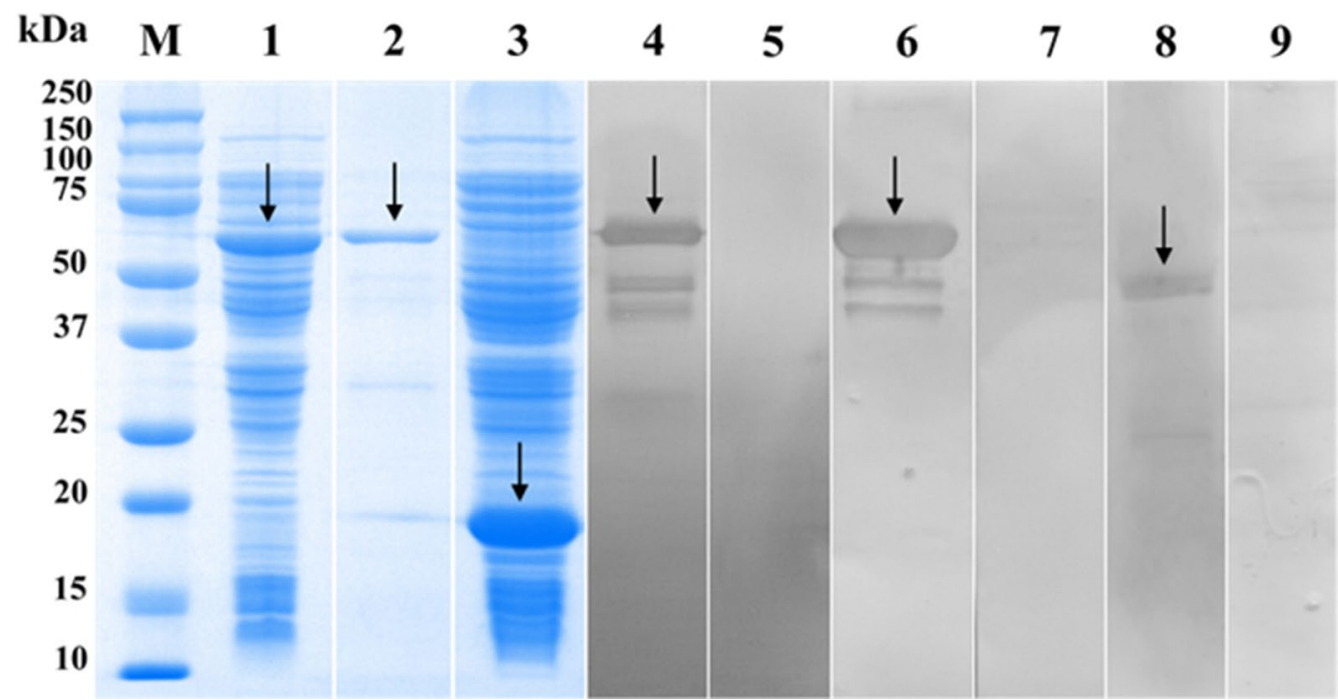

Fig. 4 Purification and western blotting of recombinant EgAnxB3 (rEgAnxB3) (a) and rEgAnxB38 (b). Lane M Protein marker, lane 1 rEgAnxB3/ rEgAnxB38 crude protein, lane 2 purified $r E g A n \times B 3 / r E g A n \times B 38$, lane $3 \mathrm{pET32a(+)} \mathrm{induced} \mathrm{by} \mathrm{isopropyl} \beta$-D-1-thiogalactopyranoside, lane 4 rEgAnxB3/rEgAnxB38 incubated with rabbit anti-rEgAnxB3/rEgAnxB38-immunoglobulin G (lgG), lane 5 rEgAnxB3/rEgAnxB38 incubated with negative rabbit serum, lane $6 \mathrm{rEgAn \times B3/rEgAn \times B38}$ incubated with serum from a sheep infected with $E$. granulosus, lane $7 \mathrm{rEgAn \times B3/rEgAn \times B38}$ incubated with serum of a healthy sheep, lane 8 crude protein of protoscoleces (PSCs) incubated with rabbit anti-rEgAnxB3/rEgAnxB38-IgG, lane 9 crude protein of PSCs incubated with negative rabbit serum IgG. For other abbreviations, see Fig. 1

liver distant from the hydatid cysts, whereas EgAnxB3 was not found in this area (Fig. 7).

\section{Phospholipid-binding bioactivity analysis}

Recombinant EgAnxB3 and EgAnxB38 were reacted with lipidosomes in the presence or absence of $\mathrm{Ca}^{2+}$ to examine their calcium-dependent phospholipid-binding properties. The mixtures were centrifuged, and the bound protein was pelleted with the precipitate and the unbound protein remained in the supernatant. In the presence of $\mathrm{Ca}^{2+}, \mathrm{rEgAnxB3}$ was observed in the precipitate rather than in the supernatant (group A). When $\mathrm{Ca}^{2+}$ was removed from the recombinant protein using EDTA, $\mathrm{r} E g A n x B 3$ largely remained in the supernatant (group B). $r E g A n x B 3$ was mainly observed in the supernatant in the absence of $\mathrm{Ca}^{2+}$ (group C). However, rEgAnxB38 was 

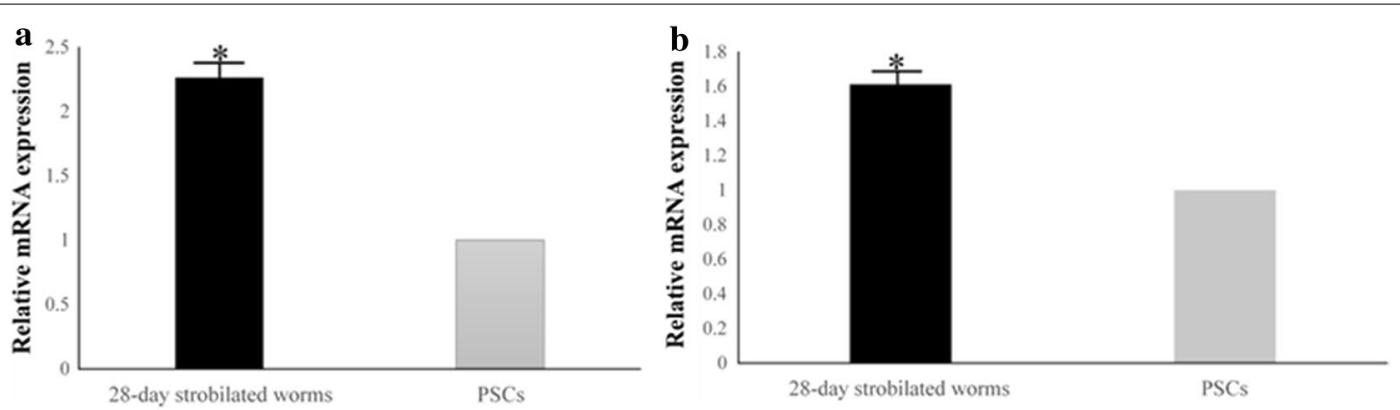

Fig. 5 Comparison of transcript levels of EgAnxB3 (a) and EgAnxB38 (b) genes in the PSCs and 28-day strobilated worms. Data are presented as the mean \pm SD of triplicate experiments. Statistically significant differences between PSCs (as the control) and 28-day strobilated worms were determined using Student's $t$-test $\left[E g A n \times B 3, t_{(4)}=14.577, P=0.00013\right.$; EgAnxB38, $\left.t_{(4)}=20.744, P=0.00003\right](* P<0.05)$. For abbreviations, see Figs. 1 and 4

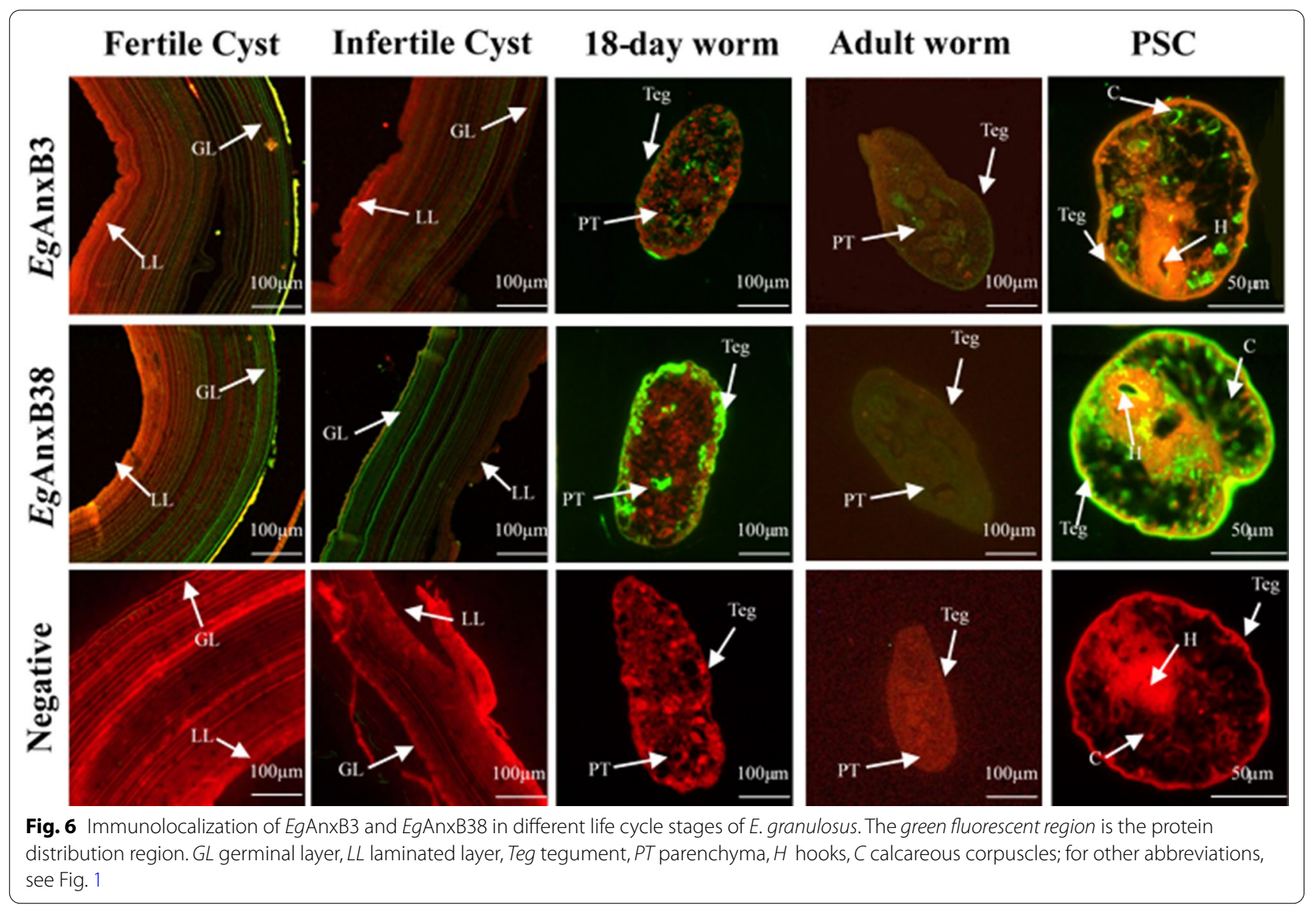

observed in both the precipitate and supernatant, regardless of the presence of $\mathrm{Ca}^{2+}$ (Fig. 8).

\section{Discussion}

The classification and nomenclature of annexin B group are confusing. Generally, parasites are invertebrates, and their annexins should be named "annexin B." However, when we screened $E g A n \times B 3$ and $E g A n \times B 38$ at the National Center for Biotechnology Information based on Zheng's research in 2013 [32], they were both called "AnxA7," which is obviously incorrect. We found that EgAnxB3 had high similarity with $T$. solium annexin B3 via sequence alignment, so we named it "EgAnxB3." However, we did not find an appropriate named parasite 

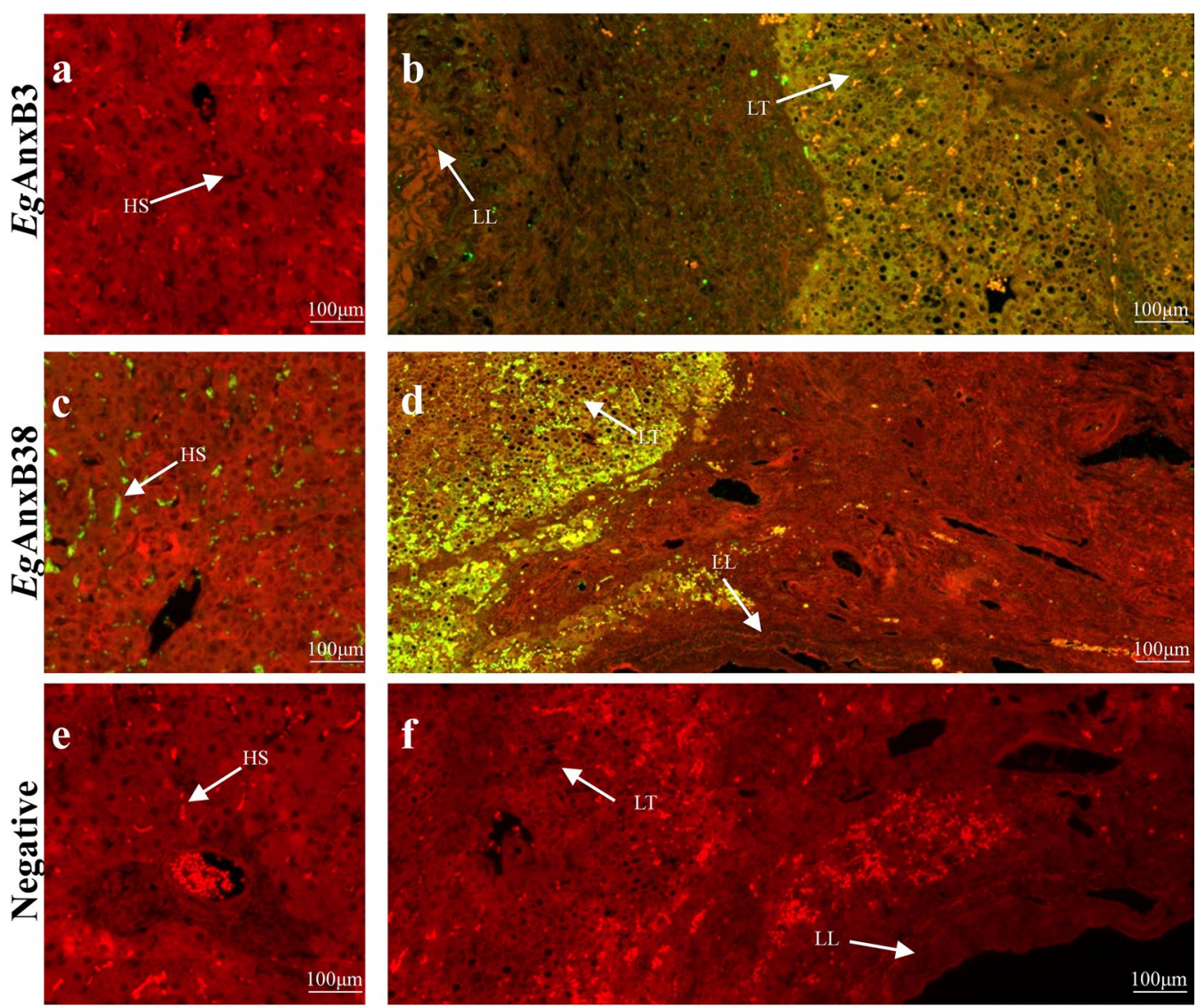

Fig. 7 a-f $I$ mmunolocalization of EgAnxB3 and EgAnxB38 in different regions of sheep liver infected with E. granulosus. a, c, e Liver tissue distant from the hydatid cysts. $\mathbf{b}, \mathbf{d}, \mathbf{f}$ Liver tissue close to hydatid cysts. The green fluorescent region is the protein distribution region. $\mathrm{HS}$ hepatic sinusoid, $L L$ laminated layer, LT liver tissue; for other abbreviations, see Fig. 1

sequence with high homology to $E g A n x B 38$. However, we found an article that named the annexins of many parasites by sequence alignment and phylogenetic tree analysis, including the annexins of E. granulosus [33]. We searched for E. granulosus annexins on the Hofmann Laboratory website (http://www.structuralchemistry.org/ annexins/seq/search.php), which showed that our naming of $E g A n x B 3$ is correct, and that the name $E g A n x B 33$ is also consistent with that previously published [24]. Therefore, we confirm here that, for E. granulosus, "EgAnxB38" should be used rather than "AnxA7."

Annexin proteins generally have four homologous repeat domains, and most of the domains contain a typical K-G-X-G-T sequence. It has been reported that the calcium-binding capacity of annexin proteins is based on their $\mathrm{K}-\mathrm{G}-\mathrm{X}-\mathrm{G}-\mathrm{T}$ sequence [33-35]. The amino acid sequence analysis showed that both $E g A n x B 3$ and EgAnxB38 have four repeat domains and contain a $\mathrm{K}-\mathrm{X}-$ $\mathrm{G}-\mathrm{T}$ sequence. The tertiary structure prediction showed that both $E g A n x B 3$ and $E g$ AnxB38 have $\mathrm{Ca}^{2+}$-binding sites. To verify the calcium-binding ability of $\mathrm{r} E g \mathrm{AnxB} 3$ and $\mathrm{r} E g \mathrm{AnxB38}$, we conducted a $\mathrm{Ca}^{2+}$-dependent phospholipid-binding assay and found that, in the absence of $\mathrm{Ca}^{2+}, \mathrm{rEgAnxB3}$ could not bind to liposomes and was found in the supernatant, while in the presence of $\mathrm{Ca}^{2+}$, $\mathrm{rEgAnxB3}$ bound with liposomes and was found in the precipitate. When EDTA was added to combine with calcium ions, $E g A n x B 3$ was again found in the supernatant, indicating that $\mathrm{r} E g \mathrm{AnxB} 3$ has $\mathrm{Ca}^{2+}$-dependent phospholipid-binding properties. However, the $\mathrm{Ca}^{2+}$-dependent phospholipid-binding properties of $\mathrm{rEgAnxB38}$ were relatively weak. $r E g A n x B 38$ was observed in both the precipitate and supernatant, regardless of the presence of $\mathrm{Ca}^{2+}$. There are two possible reasons for this. First, in this study, EgAnxB38 was expressed as an inclusion body protein, and thus possibly did not fold correctly, resulting in a lack of calcium-binding capacity. Second, regardless of the amino acid structure analysis or tertiary structure prediction, EgAnxB38 had fewer $\mathrm{Ca}^{2+}$-binding sites, resulting in an insufficient calcium-binding capacity.

The transcript levels of annexins are different at various stages of parasite development. Taenia multiceps 


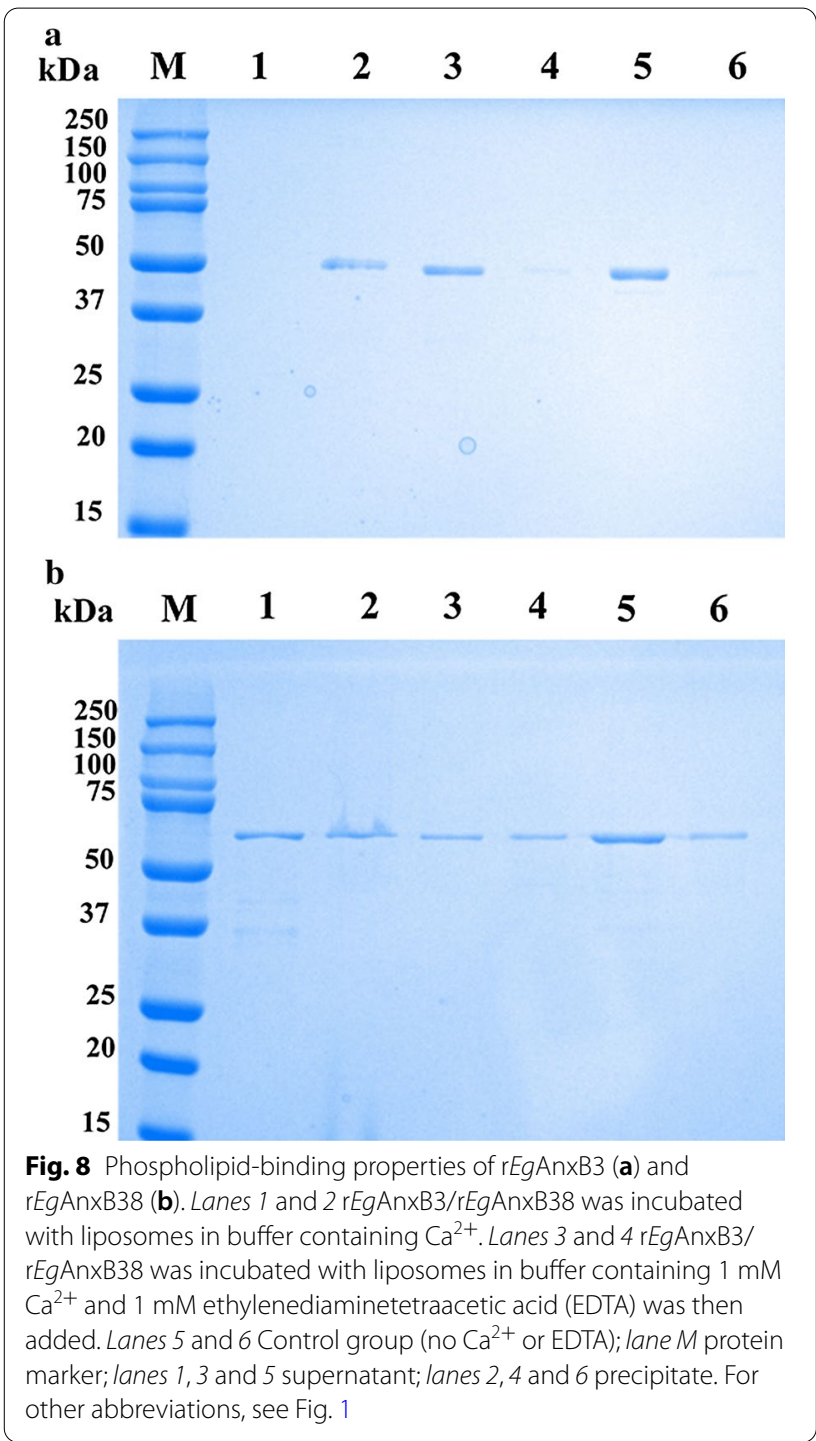

$A n x B 2$ and $A n x B 12$ show high levels of transcription in the oncosphere. The transcript levels of $A n x B 2$ and $A n \times B 12$ decreased from oncosphere to adult, while that of $A n \times B 3$ increased [34]. The transcript level of $C$. sinensis $A n x B 30$ is higher in the metacercaria stage, and lower in adult worms and eggs [36]. The transcript levels of $E g A n \times B 3$ and $E g A n \times B 38$ in the 28-day strobilated worms were higher than those of PSCs in this study. Considering that annexins can protect the parasite from the host immune system during parasitism, and that PSCs develop into adult worms in the small intestine of the definitive host [16, 17], it is suggested that $E g A n x B 3$ and $E g A n x B 38$ might play a crucial role in the process of PSC invasion of the definitive host.

The parasite communicates with the host through the tegument, and molecules distributed in the tegument participate in the host-parasite interaction, which includes excretion, nutrient absorption, and interaction with the host immune system [37, 38]. In the present study, both $E g A n x B 3$ and $E g A n x B 38$ were found to be located in the tegument and parenchyma of immature and gravid proglottids on the basis of immunofluorescence localization analysis, but $E g A n x B 38$ had a wider distribution and stronger fluorescence intensity. These results indicate that both these proteins might have a paramount role in the process of PSC invasion of the definitive host and development of the parasite, but that EgAnxB38 may possibly play a larger role than EgAnxB3 in this process. $E g A n x B 38$ was distributed in the hooks and tegument of the PSCs, while EgAnxB3 was either not distributed in the hooks and tegument at all, or at a much lower level. Considering that PSCs require the participation of the hook during development in the definitive host's intestines, it is speculated that EgAnxB38 participates in the interaction between the PSCs and the definitive host. PSCs form and mature in the germinal layer of E. granulosus [39]. EgAnxB3 and EgAnxB38 were distributed in the germinal layer, and their distribution range in fertile cysts was larger than that in infertile cysts, suggesting that they might be involved in the growth and development of PSCs.

Most annexins are cytoplasmic proteins or cytoskeletal proteins [40]; however, traces of annexins have also been found in the extracellular regions, although these annexins lack the signal peptide sequences required for extracellular secretion [41-43]. This interesting phenomenon suggests that some annexins are secreted. C. sinensis AnxB30 is a secretory protein that is involved in the interaction between the parasite and host, and affects the host's autoimmune response [36]. As a secreted protein, T. solium AnxB1 is involved in the interaction with the host inflammatory cells $[16,22,23]$. Annexins were also found to be present in the excretory-secretory product and hydatid cyst fluid of E. granulosus [6, 44]. EgAnxB33 could be detected in the hydatid fluid of E. granulosus and was located on the inflammatory cells and fibroblasts of the host-derived layer, suggesting that it might play a paramount role in the interaction between E. granulosus and the host [24]. Although EgAnxB3 and $E g$ AnxB38 have no signal peptide, in this study, they were found to be located in the hepatic sinusoids of liver tissue near hydatid cysts, indicating that they can be secreted into the extracellular areas to exert their physiological effects through non-classical secretion pathways, such as transfer via extracellular vesicles [45]. EgAnxB38 was also found to be distributed in hepatic sinusoids in the liver, which were far away from the cysts. The fact that $E g A n x B 38$ had a wider distribution in different life cycle stages of $E$. granulosus suggests that it plays a more important role in the development and interaction with 
the host. Certain excretory or secretory products of the parasite can regulate the host immune system through interaction with the host $[46,47]$. The results of this study indicate that further investigation is needed to show if $E g A n x B 3$ and $E g A n x B 38$ play a role as secreted proteins in the immune evasion of $E$. granulosus. In addition, parasite annexins have potential as drug and vaccine targets, so it is also worth exploring if $E g A n x B 3$ and $E g A n x B 38$ can be used as drug targets and vaccine candidates.

\section{Conclusions}

In conclusion, we found that both $E g A n \times B 3$ and $E g$ AnxB38 contain calcium-binding sites, and $\mathrm{r} E g$ AnxB3 has $\mathrm{Ca}^{2+}$-dependent phospholipid-binding properties. Both $r E g A n x B 3$ and $r E g A n x B 38$ could be specifically recognized by CE-positive sheep sera, indicating that they had strong immunoreactivity. The transcription level of $E g A n x B 3$ and $E g A n x B 38$ in 28-day strobilated worms was higher than that in PSCs. EgAnxB3 and EgAnxB38 were distributed in all stages of E. granulosus, and were also distributed in the liver tissues near hydatid cysts, indicating that they are secreted proteins that might play a crucial role in the development of E. granulosus.

\begin{abstract}
Abbreviations
rEgAnxB3: Recombinant Echinococcus granulosus annexin B3; rEgAnxB38: Recombinant Echinococcus granulosus annexin B38; PSCs: Protoscoleces; CE: Cystic echinococcosis; ORF: Open reading frame; HRP: Horseradish peroxidase; PCR: Polymerase chain reaction; IgG: Immunoglobulin G; EDTA: Ethylenediaminetetraacetic acid.

\section{Acknowledgements}

The authors thank Jie Xiao, Yuejun Luo, and Song Liu (Sichuan Agricultural University) for their help in performing the experiments. We also thank Lang Xiong, Ke Guan, Jiafei Zhan, and Nengxing Shen (Sichuan Agricultural University) for their constructive suggestions on the manuscript preparation. We would like to thank the native English-speaking scientists of Elixigen (Huntington Beach, CA) for editing our manuscript.
\end{abstract}

\section{Authors' contributions}

SHY participated in the design of the study, the feeding of the experimental animals, the experiments, the statistical analysis and manuscript writing. $\mathrm{HX}$ and DXD performed the experiments. HRQ contributed to sample collection and performed the experiments. XJ, HR and YGY participated in the design of the study. GXB, XY and PXR helped with the study design. All the authors read and approved the final manuscript.

\section{Funding}

This research was funded by the National Natural Science Foundation of China (grant no. 31672547) and the Key Technology R\&D Program of Sichuan Province, China (no. 2020YFN0030)

\section{Availability of data and materials}

The datasets supporting the conclusions of this article are included within the article.

\section{Ethics approval and consent to participate}

The animal study was reviewed and approved by the Animal Care and Use Committee of Sichuan Agricultural University (SYXK2019-187). All animal procedures used in this study were carried out in accordance with the Guide for the Care and Use of Laboratory Animals (National Research Council, Bethesda, MD) and recommendations of the ARRIVE guidelines (https://www.nc3rs.org. uk/arrive-guidelines). All the methods were carried out in accordance with the relevant guidelines and regulations.

\section{Consent for publication}

Not applicable.

\section{Competing interests}

The authors declare that they have no conflict of interest.

\section{Author details}

${ }^{1}$ Department of Parasitology, College of Veterinary Medicine, Sichuan Agricultural University, Wenjiang 611130, China. ${ }^{2}$ Department of Chemistry, College of Life and Basic Science, Sichuan Agricultural University, Wenjiang 611130, China.

Received: 18 November 2020 Accepted: 13 January 2021

Published online: 08 February 2021

\section{References}

1. Deplazes P, Rinaldi L, Rojas CAA, Torgerson PR, Jenkins EJ. Global distribution of alveolar and cystic echinococcosis. Adv Parasit. 2017;95:315-493.

2. Othieno E, Ocaido M, Mupere E, Omadang L, Oba P, Okwi AL. Knowledge, attitude, and beliefs of communities and health staff about Echinococcus granulosus infection in selected pastoral and agropastoral regions of Uganda. J Parasitol Res. 2018;2018:1-10.

3. Rialch A, Raina OK, Tigga MN, Anandanarayanan A, Verma MR. Evaluation of Echinococcus granulosus recombinant EgAgB8/1, EgAgB8/2 and EPC1 antigens in the diagnosis of cystic echinococcosis in buffaloes. Vet Parasitol. 2018;252:29-34

4. World Health Organization. Investing to overcome the global impact of neglected tropical diseases: third WHO report on neglected tropical diseases 2015, vol. 3. Geneva: World Health Organization; 2015.

5. Wen H, Vuitton L, Tuxun T, Li J, Vuitton DA, Zhang W, et al. Echinococcosis: advances in the 21st century. Clin Microbiol Rev. 2019;32:1-39.

6. Virginio VG, Monteiro KM, Drumond F, de Carvalho MO, Vargas DM, Zaha A, et al. Excretory/secretory products from in vitro-cultured Echinococcus granulosus protoscoleces. Mol Biochem Parasitol. 2012;183:15-22.

7. Zeghir-Bouteldja R, Polomé A, Bousbata S, Touil-Boukoffa C. Comparative proteome profiling of hydatid fluid from Algerian patients reveals cyst location-related variation in Echinococcus granulosus. Acta Trop. 2017;171:199-206.

8. Siles-Lucas M, Sánchez-Ovejero C, González-Sánchez M, González E, Falcón-Pérez JM, Boufana B, et al. Isolation and characterization of exosomes derived from fertile sheep hydatid cysts. Vet Parasitol. 2017;236:22-33.

9. Schloer S, Pajonczyk D, Rescher U. Annexins in translational research: hidden treasures to be found. Int J Mol Sci. 2018;19:1781.

10. Gerke V, Creutz CE, Moss SE. Annexins: linking $\mathrm{Ca}^{2+}$ signalling to membrane dynamics. Nat Rev Mol Cell Biol. 2005;6:449-61.

11. Chander A, Chen XL, Naidu DG. A role for diacylglycerol in annexin A7-mediated fusion of lung lamellar bodies. BBA-Mol Cell Biol L. 2007;1771:1308-18.

12. Mortimer JC, Laohavisit A, Macpherson N, Webb AAR, Brownlee C, Battey $\mathrm{NH}$, et al. Annexins: multifunctional components of growth and adaptation. J Exp Bot. 2008;59:533-44.

13. Rescher $\mathrm{U}$, Gerke V. Annexins-unique membrane binding proteins with diverse functions. J Cell Sci. 2004;1 17:2631-9.

14. Monastyrskaya K. Functional association between regulatory RNAs and the annexins. Int J Mol Sci. 2018;19:591.

15. Boye TL, Nylandsted J. Annexins in plasma membrane repair. Biol Chem. 2016;397:961-9.

16. Hofmann A, Osman A, Leow CY, Driguez P, Mcmanus DP, Jones MK. Parasite annexins-new molecules with potential for drug and vaccine development. BioEssays. 2010;32:967-76.

17. Lu Y, Wang N, Wang J, Wang K, Sun S. Expression, purification, and characterization of a novel $\mathrm{Ca}^{2+}$ - and phospholipid-binding protein annexin B2. Mol Biol Rep. 2010;37:1591-6. 
18. Pourseif MM, Moghaddam G, Saeedi N, Barzegari A, Dehghani J, Omidi Y. Current status and future prospective of vaccine development against Echinococcus granulosus. Biologicals. 2018;51:1-11.

19. Weingärtner A, Kemmer G, Müller FD, Zampieri RA, dos Santos MG, Schiller J, et al. Leishmania promastigotes lack phosphatidylserine but bind annexin $\vee$ upon permeabilization or miltefosine treatment. PLOS ONE. 2012;7:e42070.

20. Tararam CA, Farias LP, Wilson RA, Leite LCC. Schistosoma mansoni annexin 2: molecular characterization and immunolocalization. Exp Parasitol. 2010;126:146-55.

21. Leow $\mathrm{CY}$, Willis $\mathrm{C}$, Chuah $\mathrm{C}$, Leow $\mathrm{CH}$, Jones M. Immunogenicity, antibody responses and vaccine efficacy of recombinant annexin B30 against Schistosoma mansoni. Parasite Immunol. 2020;42:e12693.

22. Gao Y, Yan H, Ding F, Lu Y, Sun S. Annexin B1 at the host-parasite interface of the Taenia solium cysticercus: secreted and associated with inflammatory reaction. Acta Trop. 2007;101:192-9.

23. Yan H, Xue G, Mei Q, Ding F, Wang Y, Sun S. Calcium-dependent proapoptotic effect of Taenia solium metacestodes annexin B1 on human eosinophils: a novel strategy to prevent host immune response. Int J Biochem Cell Biol. 2008;40:2151-63.

24. Song X, Hu D, Zhong X, Wang N, Gu X, Wang T, et al. Characterization of a secretory annexin in Echinococcus granulosus. Am J Trop Med Hyg. 2016;94:626-33.

25. Khan A, El-Buni A, Ali M. Fertility of the cysts of Echinococcus granulosus in domestic herbivores from Benghazi, Libya, and the reactivity of antigens produced from them. Ann Trop Med Parasitol. 2001:95:337-42.

26. Zhan J, Song H, Wang N, Guo C, Shen N, Hua R, et al. Molecular and functional characterization of inhibitor of apoptosis proteins (IAP, BIRP) in Echinococcus granulosus. Front Microbiol. 2020;11:729.

27. Tamura K, Peterson D, Peterson N, Stecher G, Nei M, Kumar S. MEGA5: molecular evolutionary genetics analysis using maximum likelihood, evolutionary distance, and maximum parsimony methods. Mol Biol Evol. 2011;28:2731-9.

28. Xu J, Huang X, He M, Ren Y, Shen N, Li C, et al. Identification of a novel PYP-1 gene in Sarcoptes scabiei and its potential as a serodiagnostic candidate by indirect-ELISA. Parasitology. 2018;145:752-61.

29. Livak KJ, Schmittgen TD. Analysis of relative gene expression data using real-time quantitative PCR and the $2^{-\triangle \triangle C T}$ method. Methods. 2001;25:402-8.

30. Zhang Y, Wang K, Guo Y, Lu Y, Yan H, Song Y, et al. Annexin B1 from Taenia solium metacestodes is a newly characterized member of the annexin family. Biol Chem. 2007;388:601-10.

31. Choi S, Kwon S, Lee E, Kim K. Molecular cloning, functional characterization and localization of an annexin from a fish gill fluke Microcotyle sebastis (Platyhelminthes: Monogenea). Mol Biochem Parasitol. 2009;163:48-53.

32. Zheng $H$, Zhang W, Zhang L, Zhang Z, Li J, Lu G, et al. The genome of the hydatid tapeworm Echinococcus granulosus. Nat Genet. 2013;45:1168-75.

33. Cantacessi C, Seddon JM, Miller TL, Leow CY, Thomas L, Mason L, et al. A genome-wide analysis of annexins from parasitic organisms and their vectors. Sci Rep. 2013;3:1-8.
34. Guo C, Xie Y, Liu Y, Wang N, Zhan J, Zhou X, et al. Molecular characterization of annexin B2, B3 and B12 in Taenia multiceps. Genes. 2018;9:559.

35. Morgan R, Martin-Almedina S, Iglesias J, Gonzalez-Florez M, Fernandez M Evolutionary perspective on annexin calcium-binding domains. BBA-Mol Cell Res. 2004;1742:133-40.

36. He L, Ren M, Chen X, Wang X, Li S, Lin J, et al. Biochemical and immunological characterization of annexin B30 from Clonorchis sinensis excretory/ secretory products. Parasitol Res. 2014;113:2743-55.

37. Simpson AJG, Smithers SR. Schistosomes: surface, egg and circulating antigens. Curr Top Microbiol Immunol. 1985;120:205-39.

38. Dalton JP, Skelly PJ, Halton DW. Role of the tegument and gut in nutrient uptake by parasitic platyhelminths. Can J Zool. 2004;82:211-32.

39. Galindo M, Schadebrodt G, Galanti N. Echinococcus granulosus: cellular territories and morphological regions in mature protoscoleces. Exp Parasitol. 2008;119:524-33.

40. Gerke V, Moss SE. Annexins: from structure to function. Physiol Rev. 2002;82:331-71.

41. Vergnolle N, Pages P, Guimbaud R, Chaussade S, Bueno L, Escourrou J, et al. Annexin 1 is secreted in situ during ulcerative colitis in humans. Inflamm Bowel Dis. 2004;10:584-92.

42. Donnelly SR, Moss SE. Annexins in the secretory pathway. Cell Mol Life Sci. 1997;53:533-8.

43. Samia Y, Ajantha S, Egle S, Virginia C, Flower RJ, Bernhard R. Anti-allergic cromones inhibit histamine and eicosanoid release from activated human and murine mast cells by releasing annexin A1. PLoS ONE. 2013:8:e58963.

44. Aziz A, Zhang W, Li J, Loukas A, McManus DP, Mulvenna J. Proteomic characterisation of Echinococcus granulosus hydatid cyst fluid from sheep, cattle and humans. J Proteomics. 2011;74:1560-72.

45. Zhou X, Wang W, Cui F, Shi C, Ma Y, Yu Y, et al. Extracellular vesicles derived from Echinococcus granulosus hydatid cyst fluid from patients: isolation, characterization and evaluation of immunomodulatory functions on $\mathrm{T}$ cells. Int J Parasitol. 2019;49:1029-37.

46. Siracusano A, Delunardo F, Teggi A, Ortona E. Cystic echinococcosis: aspects of immune response, immunopathogenesis and immune evasion from the human host. Endocr Metab Immune Disorders Drug Targets. 2012;12:16-23.

47. Zheng Y. Strategies of Echinococcus species responses to immune attacks: implications for therapeutic tool development. Int Immunopharmacol. 2013;17:495-501.

\section{Publisher's Note}

Springer Nature remains neutral with regard to jurisdictional claims in published maps and institutional affiliations.
Ready to submit your research? Choose BMC and benefit from:

- fast, convenient online submission

- thorough peer review by experienced researchers in your field

- rapid publication on acceptance

- support for research data, including large and complex data types

- gold Open Access which fosters wider collaboration and increased citations

- maximum visibility for your research: over $100 \mathrm{M}$ website views per year

At BMC, research is always in progress.

Learn more biomedcentral.com/submissions 